\title{
Research on the Interactivity of the New Outdoor Media Advertising for the Bus Station
}

\author{
Chen Zhao, Tao Wang, Yuan-yuan Liu \\ School of Arts \& Design, Hubei University of Technology, Wuhan, China
}

\begin{abstract}
With a richer lifestyle, the consumers' attention are gradually paid into interactive and innovative, and traditional form of a single medium is already difficult to meet the psychological needs of consumers. However, most of domestic bus stop advertising is static, missing the interactive with audience, and seldom open a discussion from the view of design for new outdoor media advertising interactive. Therefore, this article combed the definition, the background, the characteristic and the advantages of interactivity of the new media advertising from the view of design study, which provided an important supplement for the design theory of the bus station advertising.
\end{abstract}

Keywords—advertising, new outdoor media, interaction, bus station

\section{公交站牌户外新媒体广告的互动性设计研究 \\ 赵晨 汪涛 刘媛媛}

湖北工业大学艺术设计学院, 武汉, 中国

\begin{abstract}
摘 要 随着人们生活方式的丰富, 消费者的注意力逐渐投向广告的创新性与互动性, 传统单一的媒介形式已很难满足消费者的 心理需求。但国内大多数公交车站牌的广告都是以静态为主, 缺乏与受众群体的互动性, 很少从设计学的角度对户外新媒体广告的互 动性展开探讨。因此, 本文从设计学的视角对户外新媒体广告的互动性概念界定及产生背景、特征和优势进行梳理, 是对研究公交车 站广告的设计理论提供重要补充。
\end{abstract}

关键词 广告, 新媒体, 互动性, 公交站牌

\section{1. 引言}

公交车作为最常用的公共交通工具之一，遍及全球。 因此, 公交站牌媒介广告是广大受众每天接触的户外广告, 所以研究公交车站的广告对广告学的健康发展有推动意 义。随着人们生活方式的丰富, 消费者的注意力逐渐投向 户外空间, 传统单一的媒介形式已很难满足消费者的心理 需求。但国内大多数公交车站牌的广告都是以静态为主, 缺乏与受众群体的互动性。近年来我国周边发达地区以及 内地北京出现的新型媒体户外广告, 纵观其成功, 无不例 外是突出了广告的互动性与创新点, 其创意引起业界专业 人士们不同凡响的欢呼声。[1]这些广告体现出的另类创意 使我感到十分有趣, 并久久徘䧃在脑海之中, 重新拓展了 我对广告内容创意的认识和看法, 也特别想引申到公交车 站的广告的互动性设计上。
从另一方面来讲, 我国现阶段对公交车站广告的互动 性研究大多是从广告学、传播学、营销学等角度进行交叉 学科的分析, 而很少从设计学和空间学的角度对公交车广 告的互动性展开探讨。[2]公交站牌媒介广告, 是针对开发、 营销市场的产品而度身打造的户外广告媒体, 是广大消费 者每天必须接触的户外广告, 在价格上与其它媒体有着得 天独厚的优势, 是电视、报纸等媒体所无法比拟的。选择 公交站牌户外广告能使所展示的广告产品直接面向广大江 城消费者, 受众群体也是极为广泛的, 与老百姓起到面对 面的交流, 就如同在有鱼的地方钓鱼一样, 使您的潜在消 费人群更直观、直接接触您的广告宣传。从媒介特性来看 我认为公交站牌户外媒介没有拘泥于电视、广播、报纸、 杂志等传统媒介单一的表达形式, 而是充分利用公交站牌 有效的空间范围, 综合运用各种媒介。因此, 从设计学的 
视角对公交车站广告的互动性概念界定及产生背景、特征 和优势进行梳理, 是对研究户外新媒体广告的设计理论提 供重要补充。在传统的公交广告站牌中展开有针对性有目 的的设计研究, 突破传统方式, 实现公交车广告站牌多元 素化的感官体验的新型户外广告。

\section{2. 理论意义与现实意义}

从理论意义来讲, 我国现阶段对公交车广告站牌研究 大多是从广告学、传播学、营销学等角度进行交叉学科的 分析, 而很少从设计学的角度对户外新媒体广告的互动性 展开探讨。因此, 本文从设计学的视角对公交车站牌新媒 体广告的互动性概念界定及产生背景、特征和优势进行梳 理, 是对研究户外新媒体广告的设计理论提供重要补充。

从现实意义来讲, 国内大多数广告站牌都是以静态的 公交车站牌为主, 但是随着户外广告的市场不断发展和成 熟, 受众购物的意识日渐理性化, 当下新媒体给户外广告 发展带来了良好的发展契机, 使得户外新媒体广告从众多 广告中脱颖而出。[3]互动性是户外新媒体广告的自身特征, 而必然也是公交车站牌广告的发展趋势, 本文以互动性为 切入点, 不仅丰富了设计人员的创作领域, 还阐释了户外 广告在新媒体环境下, 实现了传统媒体与新媒体传播之间 的相互配合, 为制作户外新媒体广告的互动性设计提供实 践价值和指导作用。新媒体互动广告可以说是广告发展的 趋势。我们已经进入了读图时代, 人们对视觉信息的需求 越来越强, 户外新媒体广告作为一种传达视觉信息的方式, 无疑需要了解人们深层次的心理需求。随着各种媒体的增 多, 观众被动接受信息的状态渐渐已无法满足人们的需求, 可以说, 传统广告的传播效果变得越来越不尽如人意。互 动新媒体广告就体现出了自身的优势, 它们可以贯穿到人 们的生活中, 其具有创意的视觉形象容易引起人们的关注, 拉近产品和消费者的距离, 使观众有效而积极的接收信息, 从而构建出一个可以提升品牌形象的平台。而公交车站又 是受众群聚集的地方, 通过等车时的间隙与户外新媒体广 告进行互动, 加深广告媒体的传播性, 因此, 研究公交车 站牌户外新媒体广告的互动性探索具有及其重要的意义。

本文试图对公交车站牌广告的互动性设计思维和设计 方法归纳总结, 并对其发展趋势作了前瞻性的探索, 提出 一些方向性的建议, 希望这些结果会为户外新媒体广告的 研究者提供一些参考, 对户外广告的健康发展有推动意义。

\section{3. 国内外研究进展}

\section{1 国外研究进展}

欧洲、美国等发达国家的户外新媒体广告发展一直处
在世界领先地位。据世界权威组织亨利中心 (HENLEY CENTRE)的调查并得出的结论是“户外新媒体广告将成为 21 世纪最具成长性和发展潜力的优异媒体”。国外新媒体的 互动性特征为户外广告带来了创新动力, 成为商家制胜的 法宝。[4][5]户外新媒体广告的发展趋向是结合数字移动设 备和互联网媒介平台的整合传播方式, 这种方式为户外广 告行业的扩张带来巨大的传播力和影响力, 为赢取经济利 益, 发挥着不可替代的作用。并且在公交站牌的设计上, 充分融入新媒体及交互式功能的公交站牌越来越多, 比如 巴西世界杯的球门公交车站、美国国家公园利用天然的石 头和木材搭建的 Yosemite 瀑布车站等。

\section{2 国内研究进展}

随着人类对健康生活方式的追求, 户外活动的增多, 户外媒体已经成为广告界的聚集的焦点, 因此, 近几年, 国内广告的增长速度远远地将电视媒体、平面媒体甩在了 后面。而且由于信息技术的发展, 国内广告牌也一改过去 呆板的平面广告牌的形象, LED、车载显示器、楼宇电梯 显示器等众多基于新技术的户外媒体运用越来越多。[6]在 此基础上, 户外媒体的创意表现也越来越广阔, 多种媒介 的组合运用, 有效的提高了广告的注目率, 向受众传递的 信息也越来越便捷、有效。户外媒体类型正面临着从平面 到立体, 从静止到流动的趋势, 从单一媒体类型逐渐被不 断丰富、细化和多元发展的组合媒介所取代。但动态且具 有交互式的人性化公交站牌还是比较少见。

\section{4. 发展动态}

随着新媒体时代的到来, 各种新技术的应用日趋成熟, 广告也随之发生了质的变化。新媒体的运用使广告的内容 变得涵盖面更广, 其形式也变得更加的多样化。[7]新媒体 与传统媒体最大的不同之处, 就是在于其可以非线性的传 播, 将时间和空间进行任意的组合, 打破了传统二维空间 的束缚, 可以营造出虚拟的三维甚至四维的空间。新媒介, 新技术，新材料的应用，使新媒体广告具有了更为开阔的 展示空间。[8]例如: 武汉江汉路 happy 站台公交车站牌前 面的雪花啤酒的广告, 打破了传统的平面广告, 虚拟出三 维空间, 在广告牌玻璃中种上小麦, 然后结合二维的字体 与图案, 打上麦粒色黄色的灯光, 让整个广告牌非常夺目, 有效的将广告牌从单项静态的形式向双向的多维的多种感 官并用的信息综合体转变。这时候, 互动形式就显得极为 重要, 它可以让观众参与到信息的传播中去, 从被动的接 受变成主动的想要参与体验。由此可见, 设计的理念更新, 投放形式多元化发展是广告行业前进的必经之路, 互动性 
则是户外广告发展的一个必然趋势。

\section{1 传播技术更智能化}

科技发展的迅速, 带来了许多新的传播技术手段, 使 户外新媒体广告的传播变得更加智能化。目前, 这些技术 已经广泛应用于各个领域, 其超大空间的无限创意, 增加 了户外新媒体广告与受众之间的沟通, 受众不仅可以和广 告信息进行互动, 还可以透过体验了解产品及品牌理念, 实现了真正意义上的双向传播。因此, 从营销的角度来讲, 科技的发展让户外新媒体广告变成了各式各样的智能化销 售平台。此外, 科技的进步让户外测量变得有突破的可能, 即测量受众与户外新媒体广告之间的互动情况。可以通过 植入调查系统, 利用触控电子屏等技术渠道检测受众与广 告之间交流的频次, 结果更有助于准确定位广告设计的媒 介选择和受众倾向, 提高传播效率。由此可见, 传播技术 的革新, 改变了消费者的生活形态, 为户外新媒体带来了 新的契机和广告播放的智能化。

\section{2 媒介整合更多元化}

媒介整合的时代已经到来, 这是我们都能感受到的变 化。科技的发展让我们把户外、技术、互联网等不同领域 结合在一起, 成为各种信息交融落地的多元化平台, 形成 一个良性的循环传播体系。从目前的媒介环境来看, 可以 说媒介整合有两大趋势: 一是传统媒介充分利用新的传播 技术和传播方式, 主动或被动地谋求多媒体化经营, 以求 实现自身与依托于网络等各类媒介之间的整合; 二是产生 一种全新的媒介形式, 力求打破传统媒介与新媒介两者之 间的界限, 不再仅仅是各类不同形式的媒体之间的资源共 享和分工协作, 而是一种或几种整合了以往的媒介形态, 改变了以往媒介对人类感官功能部分的延伸缺陷, 充分利 用媒体的多样化重新整合媒介形态, 这种媒介形态有着极 强的互动性和参与性, 以网络作为载体平台和发行平台, 最终通过各类不同的端设备呈现出来, 是户外新媒体广告 开辟新市场、拓展利润空间的创新模式。媒介整合突破了 传统媒介无法互动交流的思路, 合理利用多元化的媒介形 态对广告主和消费者来说都是一种增值。善于运用传统媒 体和新媒体融合产生的综合效应, 有效的将二者整合互补, 并且拓展现有的传播资源, 实现与消费者之间的互动关系, 这对企业的整合营销传播发展具有深远的影响。

综上所述, 户外新媒体广告的互动性设计发展潜力巨 大, 互动性特点的运用在未来的传播中, 必然是综合各方 面的因素, 在全方位的思考与探索中逐步完善。随着科技的 发展, 户外新媒体广告将面临更大的发展机遇, 是传统户
外广告发展的生存之道, 我们有理由相信其在稳步发展中 将迎来更为广阔的天地。

\section{5. 多视角分析}

从传播学角度来说, 提高广告的互动性设计也是现代 广告学发展的必然趋势, 当广告主与受众群体产生有效的 互动性, 当消费者开始参与广告, 从传统的被动变为有效 的互动, 不仅加深了广告在消费者心中的形象定位, 也能 使之有效的记住广告品牌,

从现代设计学的角度来说, 提高广告的互动性设计可 以从视觉体验、嗅觉体验、触觉体验以及听觉体验入手。 带入受众群体进入多感官体验的广告互动时代。

从设计学的角度分析, 公交车站广告的互动性设计有 效的以非线性的传播, 将时间和空间进行任意的组合, 打 破了传统二维空间的束缚, 可以营造出虚拟的三维甚至四 维的空间。新媒介, 新技术, 新材料的应用, 使新媒体广 告具有了更为开阔的展示空间。将空间变成一种媒介, 打 破传统静态的传播方式, 让广告变得更加立体跟多样化。

新型的公交车站广告有着高效、环保的特征。它不仅 能够保护环境, 节约资源, 节省成本, 还能够更加有效的 传播了广告信息。在使商家的利益得到最大化的同时, 也 为了社会公益做出了贡献。在经济飞速发展的现今, 新媒 体广告的互动性传播优势变得更加显著, 得到了人们的喜 爱和认可。因其远胜于传统广告的传播特征, 加快了它的 发展速度, 扩大了发展空间。此外, 传统的户外广告大多 制作粗粘, 内容知识无法满足日益增长的审美情趣, 甚至 影响了城市的整体形象, 这迫使政府增加户外广告和训练 强度, 因此, 许多传统的户外广告公司的生存面临严重的 威胁。而这时新媒体广告也应运而生, 其尽可能多的信息 传播方式受到客商喜爱广告越来越显著的传播优势, 使其 得到了社会的肯定，进而得到了全面快速的发展空间。

当视觉、听觉以及触觉无意识的接受到信息信号时, 若没有及时引起人的注意, 那么瞬时记忆便会很快消失掉, 也不会在大脑海马体中留下任何印象也就是所谓的短时记 忆; 若及时引起人的注意, 甚至刺激到大脑海马体, 海马 体便会自动储存并赋予意义, 容量及保持时间图像记忆最 长可达到几周甚至几个月之久, 以便人类随时提取记忆。 “一闪而过”的瞬间能够引起消费者的注意力成为户外广告 成功与否的衡量标准, 于是商家们开始绞尽脑汁的想如何 制造才能刺激消费者去记忆。人天生就具有对新鲜、新奇 东西的好奇心, 正如娱乐性质的媒体总是拿前卫新鲜甚至 无论正面与负面、只要够爆炸够吸引的消息吸引大众的关 注焦点一样, 虽传播的信息不全是积极向上的, 但是倍受 关注的速度绝对是一流快的。户外广告的商家、设计师们 
受到启发, 开始无时无刻的寻找新的表现方法来推销自己 的产品。突破, 迫在眉睫; 入口, 就在“创新”二字上。而 单单 的只是局限在“创新”二字上也很难有很大的上升空 间, 只有将创新与互动二者相结合, 才能使公交站广告更 加新颖夺目。

\section{6. 结论}

目前公交车站牌互动性广告虽然仍未完全取缔传统媒 体广告牌, 但在国外各主要城市以及中国少部分城市, 它 已成为有效的广告媒体, 传统公交车站牌广告即使使用更 多的预算也无法达到新型广告公交车站的互动性传播效 果, 传统户外广告已逐渐退出业界舞台。有人说: 在户外 做有创意的广告, 加之日新月异的科技手段的运用, 可以 使平静的街道化为缤纷的剧场。以媒体、创意为依托的户 外新媒体广告, 值得每一位从事广告、媒介行业的人士关 注、学习和投入。

\section{参考文献(References)}

[1] Verolien Cauberghe, Patrick De Pelsmacker, Wim Janssens, "Simultaneous exposure to a program and advertising content in an interactive context: Perceptual and semantic interference and reinforcement," Journal of Business Research, Vol. 63,
Issues 9-10, September-October 2010, pp. 972-978.

[2] Chulei Xia, "Research on the interactive advertising and the image of the city brand communication under the background of new media," Journalism \& Communication, Vol. 7, pp. 90-91.

[3] Xueqin Wang, "Social interaction form in new media art field," Journal of Huaqiao University (Philosophy \& Social Sciences), 2013, Vol. 2, pp. 156-160.

[4] Qinqin Xie, "Research on the new media interactive advertising," Modern Decoration (Theory), 2012, Vol. 3, pp. 138-139.

[5] Dingyi Fan, "Research on the interactive creativity of the new media outdoor advertising," Packing World, 2014, Vol. 1, pp. 21.

[6] Mei Shu, "The clever environment medium-a kind of novelty communication way," Anhui Architecture, 2007, Vol. 2, pp. $11-12$

[7] Huiling Wang, "Experience of the era of environmental media advertising innovation," China Advertising, 2008, Vol. 10, pp. $118-120$

[8] Yan Chen, "The development of China's new outdoor media," Admen, 2008, Vol. 12, pp. 186-187. 